\title{
Contrôle d'ambiance par rideaux d'air
}

\author{
François-Xavier Blanchet ${ }^{1}$, Claude Cassin $^{2}$, Yvan Gouriou ${ }^{1}$, Sanjeev Gupta ${ }^{1}$, \\ Pierre Maubert ${ }^{2}$, Michel Pavageau ${ }^{1}$, Clande Rey ${ }^{2, a}$ et Camille Sollied ${ }^{1}$ \\ 1 École des mines de Nantes, UMR 6144 CNRS-EMN-Université de Nantes, Département Systèmes Énergétiques \\ et Environnement, 4 rue Alfred Kastler, BP 20722, 44307 Nantes Cedex 3, France \\ 2 Université Paul Cézanne d'Aix-Marseille, IUT de Saint Jérôme, Département GTE, 142 traverse Charles Susini, BP 157, \\ 13388 Marseille Cedex 13, France
}

Reçu le 7 mars 2005

\begin{abstract}
Résumé - Les rideaux d'air sont des jets plan traversants conçus pour isoler deux volumes adjacents communicants. De tels systèmes sont communément utilisés dans le but de minimiser les transferts de masse ou de chaleur entres deux zones tout en conservant une libre circulation des personnes et des engins entre ces mêmes zones. Des installations expérimentales ont été conçues pour étudier plusieurs configurations de rideaux d'air et déterminer la meilleure efficacité pour préserver l'énergie ou pour confiner les fumées dans le cas de feux en tunnels routiers. L'analyse a porté principalement sur la dynamique de l'écoulement. Deux configurations composées d'un ou deux rideaux de jet d'air simple ou double ont été comparées. Elles ont été réalisées pour faciliter les mesures vélocimétriques par images de particules (PIV).
\end{abstract}

Mots clés : Rideau d'air / soufflerie / impact de jet / confinement d'ambiances

Abstract - Air curtains are plane air jets blown across openings so as to isolate from each other two adjacent volumes. Such apparatus are commonly used in applications where it is sought to minimise heat or mass transfers between two areas while it is necessary to keep free the way for people or material from one area to the next one. Experimental facilities were designed to investigate various air-curtain arrangements with the aim to work out efficient solutions for energy saves or smoke confining in case of fire in a road tunnel. In the first stage, the emphasis was put on flow dynamics. The different configurations considered in this work include systems made of one or two curtains of single or double jets. They were specifically designed to perform measurements using Particle Image Velocimetry (PIV).

Key words: Air curtain system / tunnel / impinging jet / ambience confining

\section{Introduction}

Un rideau d'air est constitué par un jet d'air plan vertical descendant qui réalise une porte immatérielle séparant deux milieux ambiants. Le rideau d'air limite les échanges d'énergie et de masse entre les deux ambiances sans faire obstruction aux manipulations ou passages de personnes et d'engins. Ce principe est actuellement très utilisé en protection thermique des entrées de magasins et lieux publics, on le retrouve dans les dispositifs de présentation de produits d'alimentation réfrigérés et en protection des manipulateurs sous hotte d'aspiration.

\footnotetext{
a Auteur correspondant : crey@L3m.univ-mrs.fr
}

Le travail réalisé répond à deux objectifs :

- Renforcer l'« étanchéité » du rideau d'air.

- Réaliser un système de confinement cellulaire par rideau d'air, applicable aux tunnels routiers, couloirs de dégagement, sas de halle de stockage...

Ces deux objectifs donnèrent lieu à 3 études techniques de conception et réalisation pour les étudiants du département GTE de l'IUT de Marseille.

L'exploitation a été ensuite faite à l'école des mines de Nantes, au cours de projets d'élèves ingénieurs du Département Systèmes Énergétiques et Environnement. Une soufflerie spécifique pour application à la ventilation 
de tunnels routiers a alors été conçue et réalisée à l'école des mines de Nantes dans le cadre d'une thèse.

\section{Le rideau d'air « jet simple - double flux »}

\subsection{L'étude technique}

Il s'agit de l'étude technique menée pendant deux ans par les étudiants d'IUT, pour mettre en œuvre un procédé de séparation d'ambiance entre un milieu ambiant intérieur (A) et un milieu ambiant extérieur (B). La séparation est réalisée par un rideau d'air constitué d'un jet plan vertical descendant unique réunissant deux flux, l'un en provenance du milieu (A), l'autre en provenance du milieu (B) et impactant sur un sol horizontal plan.

Le principe théorique est le suivant : les débits respectifs des flux, A (issu du milieu ambiant A) et B (issu du milieu ambiant $B$ ), sont réglés de telle sorte que les échanges de masse entre les ambiances A et B soient minima (Fig. 1). Par ce procédé, le jet se comporte comme un jet plan unique classique dont on connaît les propriétés dynamiques par la théorie des jets. Si les fluides A et B sont identiques les échanges minima de masses sont localisés dans le plan de symétrie dynamique du jet.

Les propriétés du jet sont :

\section{Propriété 1}

«Il y a entraînement de masse par le jet ». L'entraînement de la masse aux frontières du jet se fait de part et d'autre du plan de symétrie XX' dans les zones de mélange maximal du jet (définies sur le schéma figure 1, indication pour le milieu A). Ainsi, dans le demi-espace A le jet entraîne et se mélange aux masses de fluide en provenance de la même ambiance $\mathrm{A}$ adjacente à cet écoulement. De même l'écoulement dans le demi-espace coté B, puisé à l'origine dans l'ambiance $\mathrm{B}$, ne se mélange qu'avec l'ambiance $\mathrm{B}$ dans la zone de mélange maximal (cf. Fig. 1).

\section{Propriété 2}

«les contraintes, flux de masse et de chaleur sont nuls en moyenne sur l'axe de symétrie du jet en écoulement établi ». Bien qu'en théorie, il n'y ait localement pas d'échange de part et d'autre du plan de symétrie du jet établi, ces mélanges existent par transport des flux issus des zones d'établissement du jet mais leur importance diminue progressivement vers l'aval. Les échanges se font alors principalement à l'impact sur le sol, ils sont très inférieurs en intensité par rapport à ceux qui sont localisés dans la zone de mélange maximal, précédemment décrite.

Il en résulte que puisque la seule zone d'échange possible entre les fluides A et B est le plan de symétrie XX', la séparation d'ambiance est optimisée en comparaison à tout autre procédé préservant l'énergie et une ventilation à puisage unique dans l'ambiance à préserver.

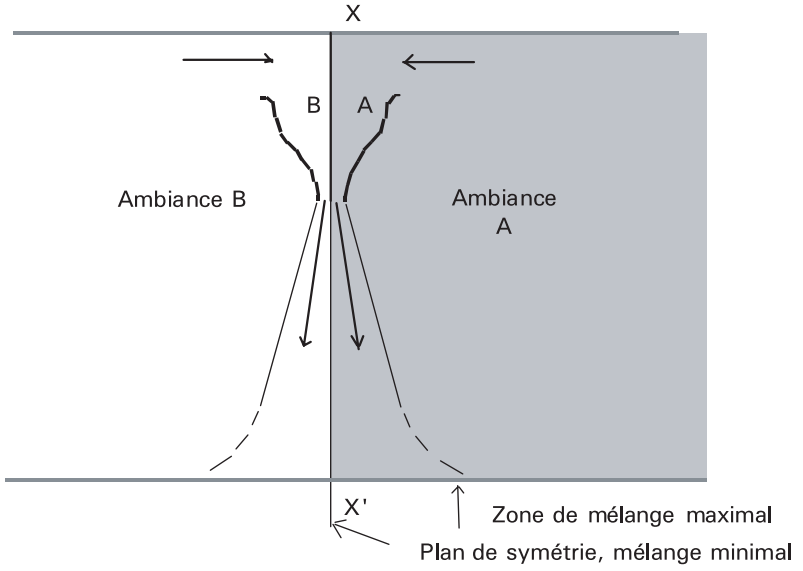

Fig. 1. Schéma de principe du rideau d'air à jet unique double flux.

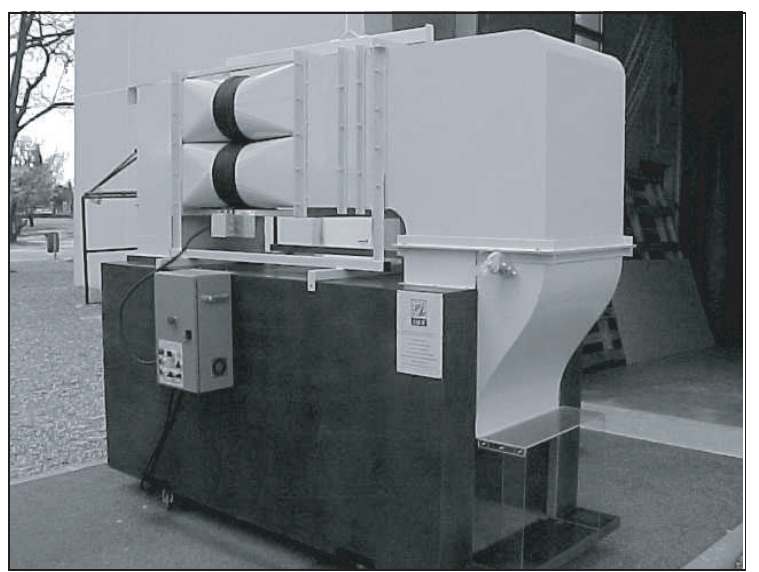

Photo. 1. La maquette prototype.

La maquette prototype d'étude réalisée (photo 1) permet de qualifier le procédé en comparant le jet double flux et le jet simple flux. Les schémas d'ensemble et de fonctionnement comparatifs sont donnés sur la figure 2 .

\subsection{Méthodologies expérimentales}

L'analyse expérimentale a permis de qualifier et de quantifier l'efficacité du jet vertical descendant « rideau d'air », pour deux configurations : Le rideau d'air classique dit à flux unique et le rideau d'air double flux, analysés de façon comparative pour évaluer les améliorations apportées.

L'efficacité du jet double flux en séparateur d'ambiance a été testée par mesure du temps de résidence : temps de décroissance de la concentration d'un traceur dans la cellule à protéger (Fig. 3).

Cela revient à quantifier le taux d'échange de masse au cours du temps : efficacité du rideau d'air. Pour les trois temps décrits ci-dessous on mesure la concentration moyenne en éthane au sein de la cavité au cours du temps.

- La cavité est étanchée par une porte matérielle pendant le temps de remplissage. 


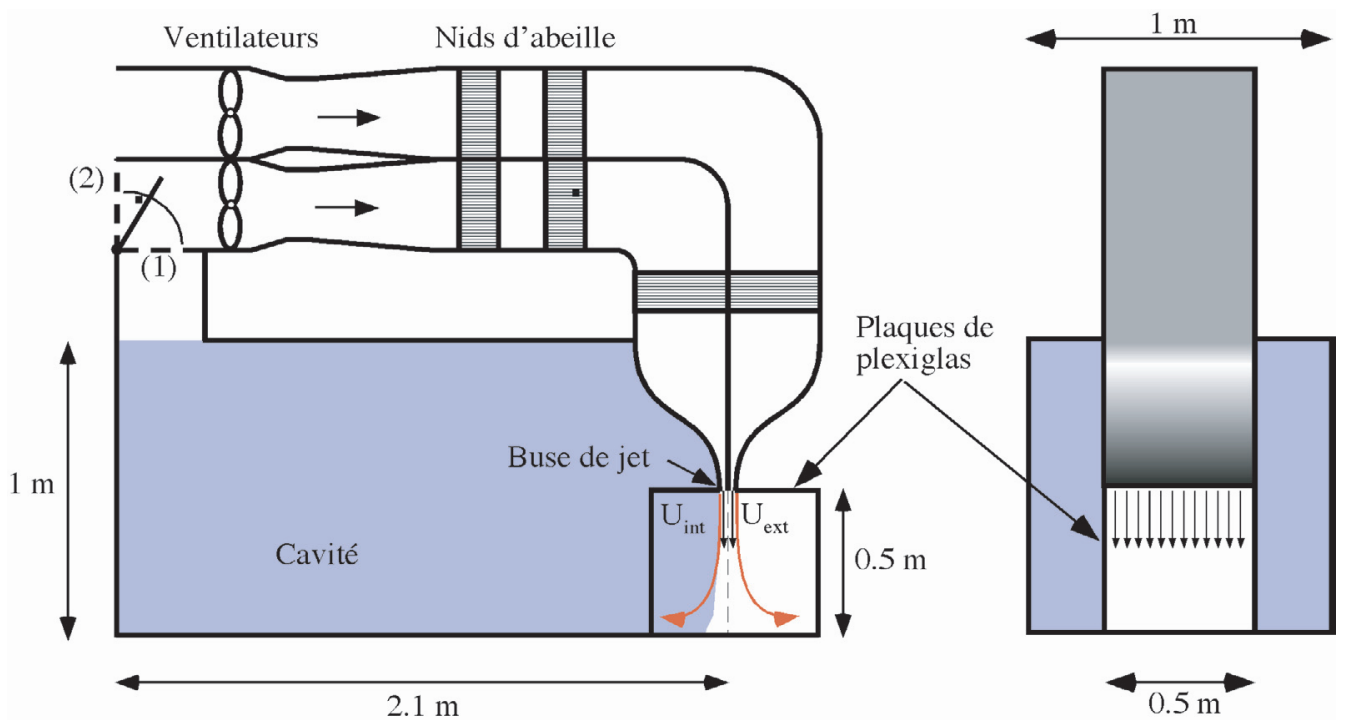

Fig. 2. Maquette prototype d'étude. Jet double flux avec reprise : Vanne en position (2).

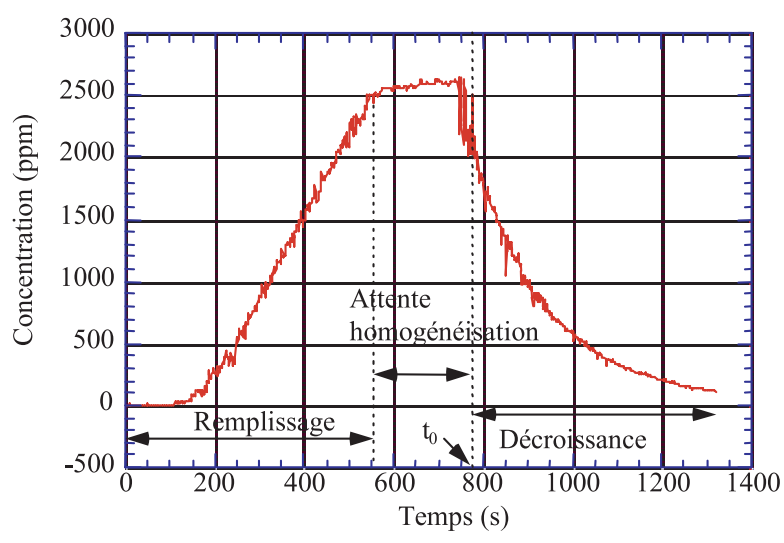

Fig. 3. Mesure du temps de résidence.

- On laisse le mélange s'homogénéiser (temps de stabilisation).

- Le rideau d'air est mis en fonctionnement en même temps que la porte est retirée «instantanément », on mesure alors le temps de décroissance.

Les temps de décroissance obtenus pour diverses configurations sont comparés, en figure 4, ce qui démontre l'efficacité du système «double flux avec reprise »: Plus le temps de résidence est long, plus le système est efficace.

La technique PIV (Particle Image Velocimetry) complète l'analyse : Il s'agit d'une méthode de mesure qualitative et quantitative, par visualisation et vélocimétrie à imagerie laser (Fig. 5) :

- Des masses d'air sont ensemencées de fines particules qui servent au traçage de l'écoulement.

- Un plan lumineux est réalisé au moyen d'une source laser dont le rayon est diffusé dans le plan par un prisme ou un disque tournant équipé de facettes miroir.

- Le traitement d'image associé donne soit une visualisation instantanée des concentrations de particules

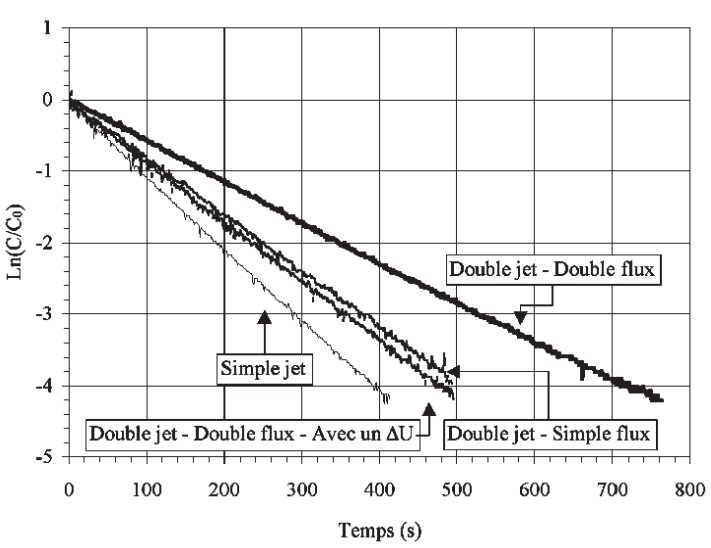

Fig. 4. Décroissance de la concentration au cours du temps.

dans le plan, soit le champ instantané des vecteurs vitesse symbolisés par des flèches (Fig. 5b).

- Le traitement ultérieur des données permet de tracer les profils de vitesse dans différents plans en fonction de l'éloignement de la bouche de jet.

\subsection{Intérêt du jet double flux avec reprise pour l'application aux bâtiments industriels}

Le système décrit permet de minimiser les échanges de masse ou de chaleur entre deux ambiances identifiées. Si l'ambiance B (Fig. 1) est une cellule d'habitation, de stockage, de circulation ou de production, dont l'accès se fait par une porte réalisée par le «plan de symétrie » dudit « rideau d'air à jet unique double flux », l'ambiance $B$ est mieux protégée dans son confinement énergétique et matériel du fait du mélange minimal de masse entre A et B. 


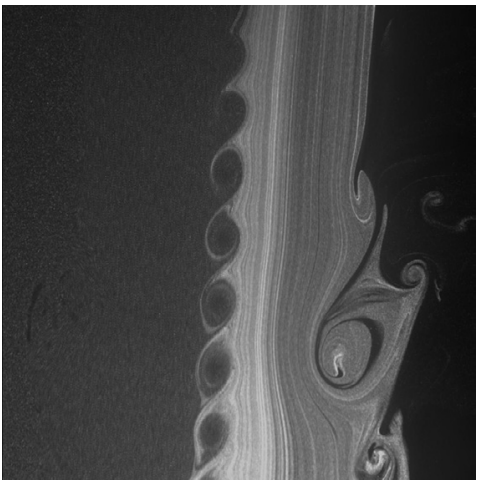

a. Analyse qualitative : Visualisation.
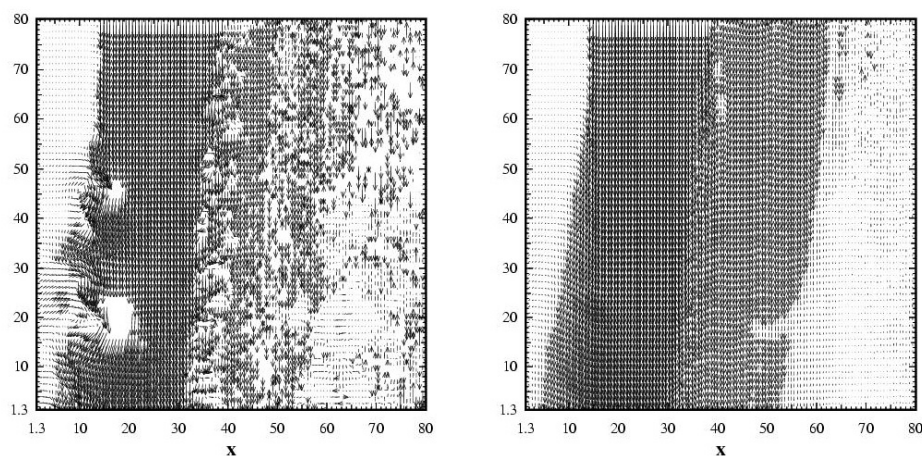

b. Analyse quantitative : Champ des vitesses instantanées, champs des vitesses moyennes.

Fig. 5. Analyse par PIV.
- Conditionnement d'une ambiance. Les échanges avec le milieu extérieur sont minimisés, mais existent, ils seront pris en compte dans le contrôle du renouvellement d'air de la cellule B.

- Sécurité incendie. En cas de début d'incendie dans la cellule B, l'ambiance B n'est pas renouvelée suffisamment, elle s'appauvrit en oxygène, ce qui empêche une propagation rapide des foyers. La porte fonctionne en coupe feu qui peut être traversé pour l'évacuation des personnes et pour l'accès du personnel de lutte contre l'incendie.

\section{La ventilation cellulaire}

\subsection{L'étude technique}

Il s'agit de l'étude technique menée par les étudiants d'IUT, pour mettre en œuvre un procédé de ventilation cellulaire dont le principe a été testé sur maquette (système des gaines et trappes), et qui consiste à ventiler un tunnel ou couloir de grande longueur par une succession de jets transversaux et reprise d'air en plafond. (Figs. 6 et 7 ).

Le fonctionnement est double : fonctionnement ventilation-aération (Fig. 7) et fonctionnement accidentel (Fig. 8), où la zone sinistrée est isolée par une ventilation court-circuitée sur la cellule concernée.

La maquette des gaines de ventilation avec trappes et volets permettant les deux types de fonctionnement est présentée en photo 2.

\subsection{Exploitation du jet double flux avec reprise partielle, en ventilation cellulaire, par visualisation et vélocimétrie à imagerie laser}

Une soufflerie a été construite à l'école des mines de Nantes pour approfondir l'étude du système de ventilation cellulaire, sous forme d'une maquette de tunnel. La conception est liée aux techniques de mesure utilisées, par

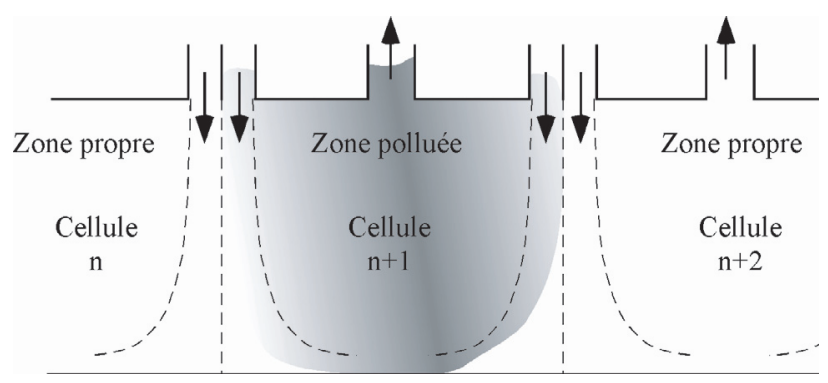

Fig. 6. Schéma de principe de la ventilation cellulaire.

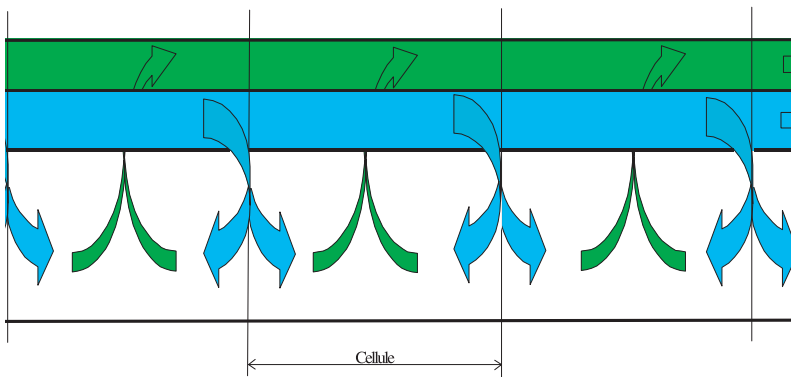

Fig. 7. Fonctionnement en mode « ventilation ».

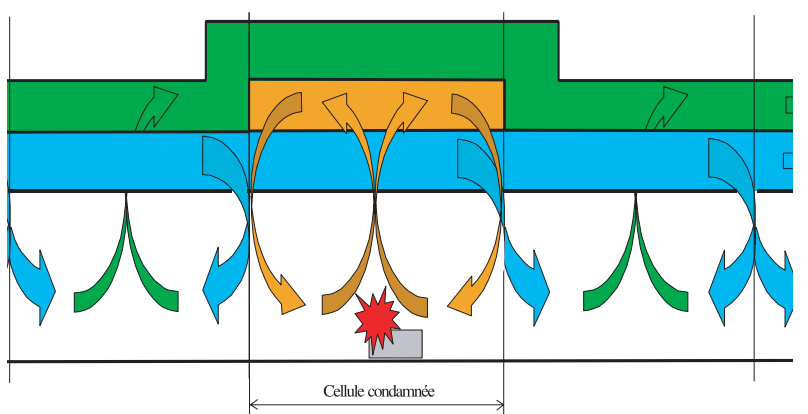

Fig. 8. Fonctionnement en mode « accident ».

PIV (Particle Image Velocimetry), avec saisie et traitement d'images de l'écoulement ensemencé de fines particules, éclairé par un plan lumineux laser. L'intérêt ici est d'identifier séparément les deux flux réalisant le rideau d'air. 


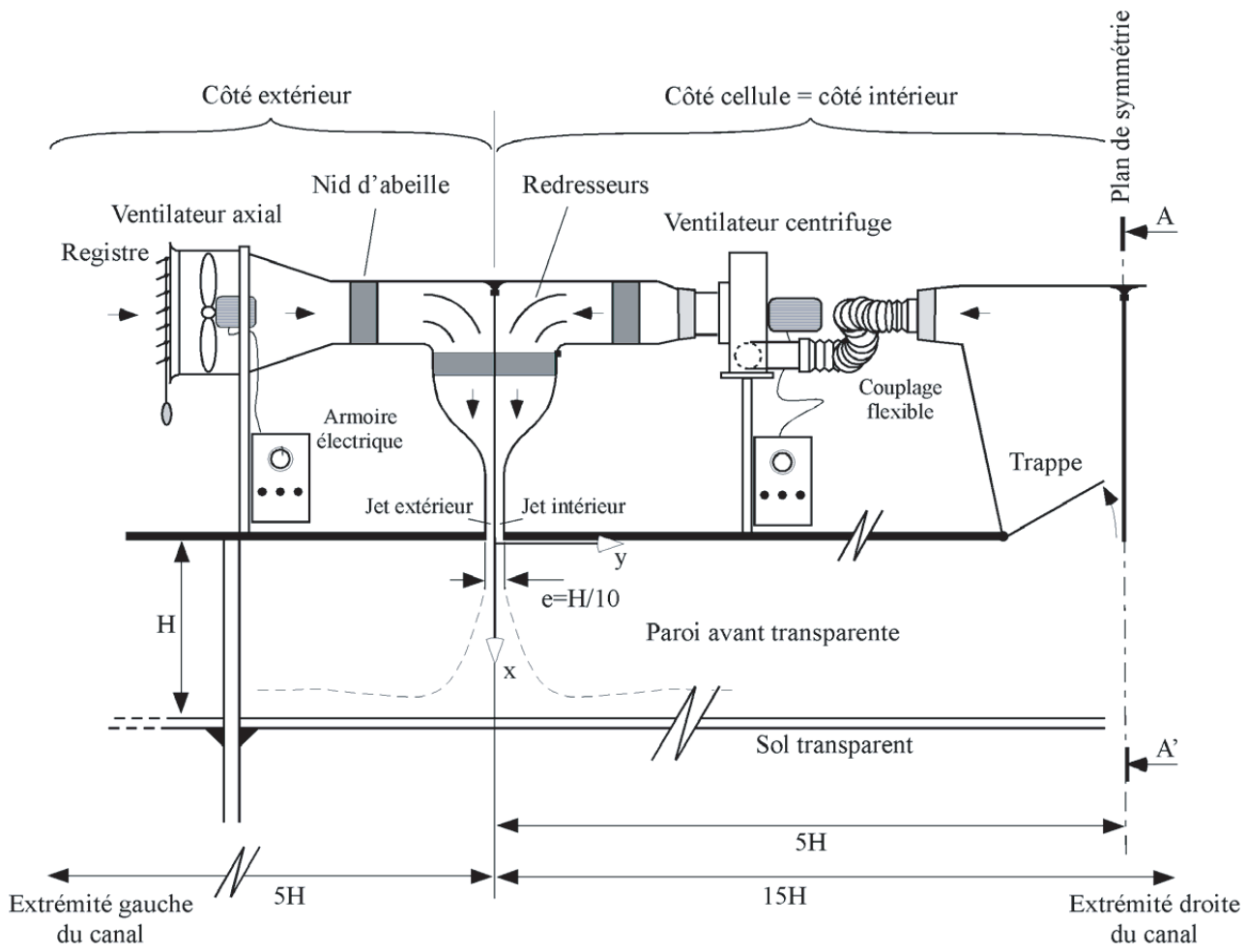

Fig. 9. Schéma de principe du banc d'essais (moitie de gauche par rapport à la photographie).

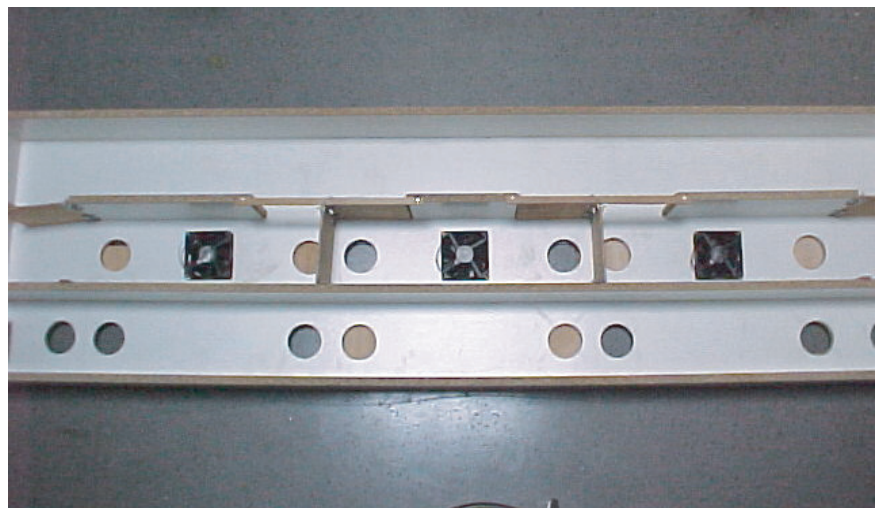

Photo. 2. La maquette des gaines de ventilation.

Cette technique PIV permet par exemple d'accéder rapidement à une estimation des champs d'énergie cinétique turbulente (Fig. 10). Le traitement des champs de vitesse, à l'impact, dans des plans parallèles au plan de la buse de soufflage a par ailleurs conduit à une première caractérisation de la cinématique des jets en présence et à une étude statistique des structures présentes dans cette région de l'écoulement. Les mesures de décroissance de concentration d'un gaz traceur au sein des cellules de confinement ont confirmé la supériorité du jet simple double flux dans le cas le plus critique du soufflage de jets droits de rapport d'ouverture $\mathrm{H} / \mathrm{e}=10$. La constante de temps de ce type de dispositif est de 30 à $40 \%$ plus importante que celle d'un dispositif classique, à volume confiné identique et à même énergie consommée par les

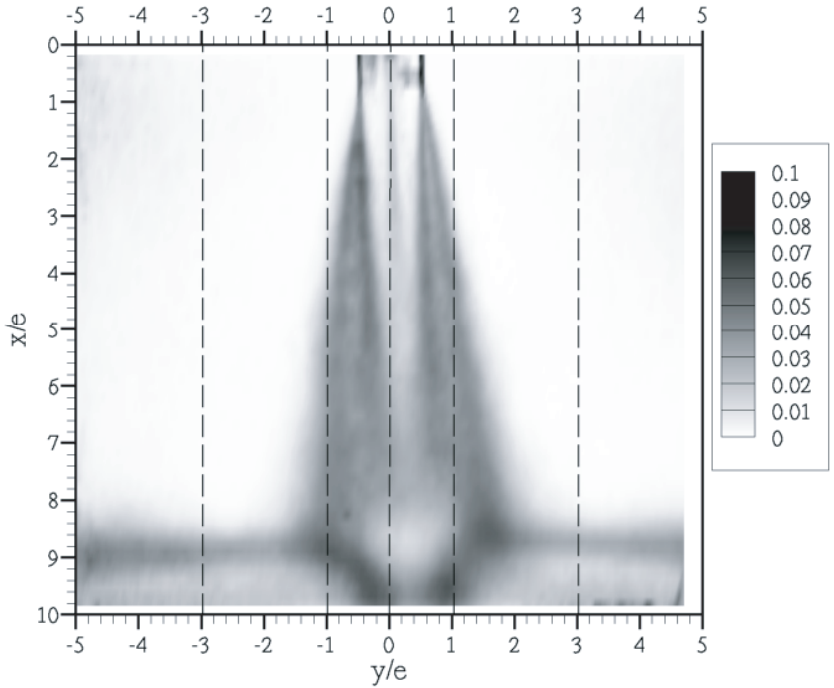

Fig. 10. Champ d'énergie cinétique turbulente $\left(\sigma_{U}^{2}+\sigma_{V}^{2}\right) / U_{0}^{2}$ pour un jet double.

systèmes de ventilation. On confine donc plus longtemps avec un jet simple double flux.

Nous avons réalisé des mesures synchrones de pression différentielle instantanée à l'aide d'un capteur piezzorésistif multivoie. Les prises de pression sont affleurantes à la paroi (Fig. 11). Elles ont un diamètre interne de 8/10 mm. Percées le long de l'axe du tunnel, elles sont au nombre de 31 , réparties symétriquement de part et d'autre du plan de symétrie de la buse. 


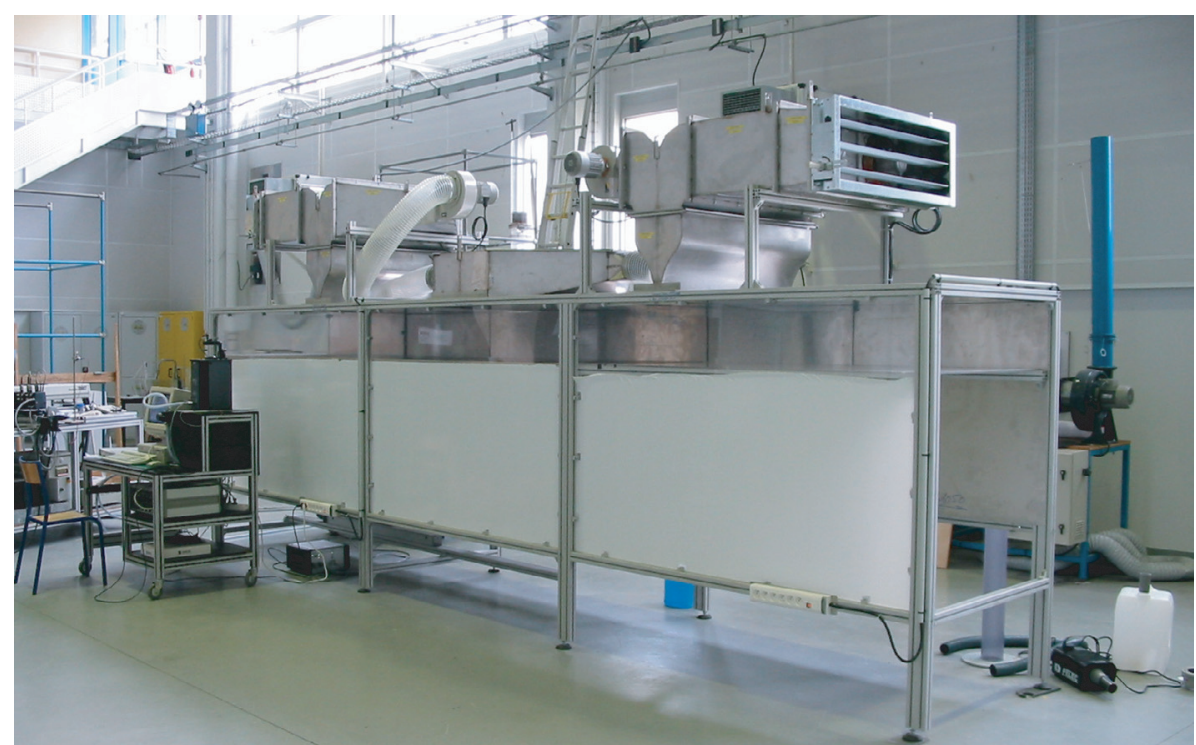

Photo. 3. Le banc d'essais ventilation cellulaire de l'école des mines de Nantes.

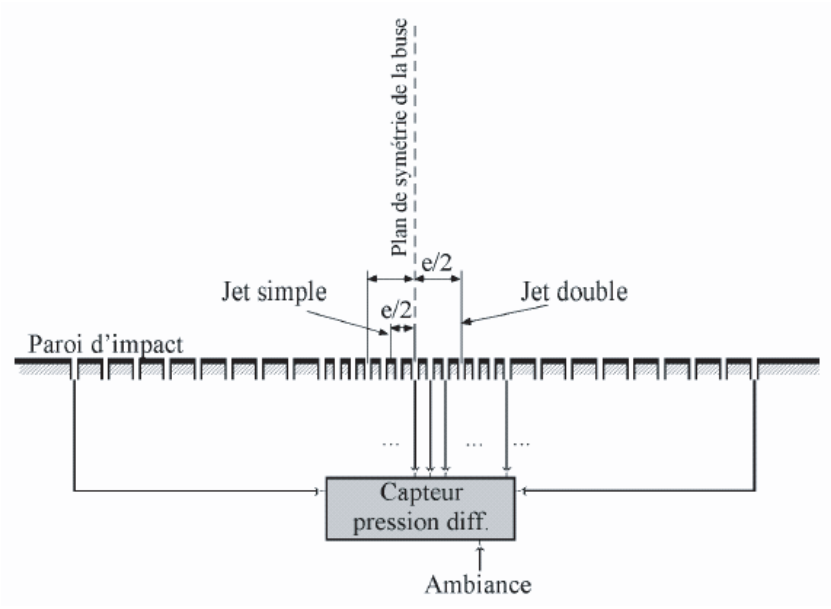

Fig. 11. Schéma du dispositif de mesure des profils de pression pariétale à l'impact.

En outre, comme le montrent des mesures de pression à l'impact de différents rideaux testés sur ce banc, l'efficacité des systèmes double flux se trouve renforcée de fait par la boucle de recirculation présente sur ce type de dispositif (Fig. 12).

\subsection{Intérêt pour l'application aux ouvrages}

L'intérêt principal est la capacité d'isoler une cellule en court-circuitant sa ventilation, il n'est plus amené d'air frais, d'où l'étouffement du foyer en cas d'accident. Les fumées excédentaires sont évacuées par les cellules voisines dont le fonctionnement n'est pas altéré. En cas de dégagement toxique les cellules voisines peuvent être court-circuitées à leur tour pour ralentir la propagation et permettre l'évacuation. Le principe « porte immatérielle » reste très précieux pour la circulation des personnes et des engins, évacuation comme accessibilité secours.

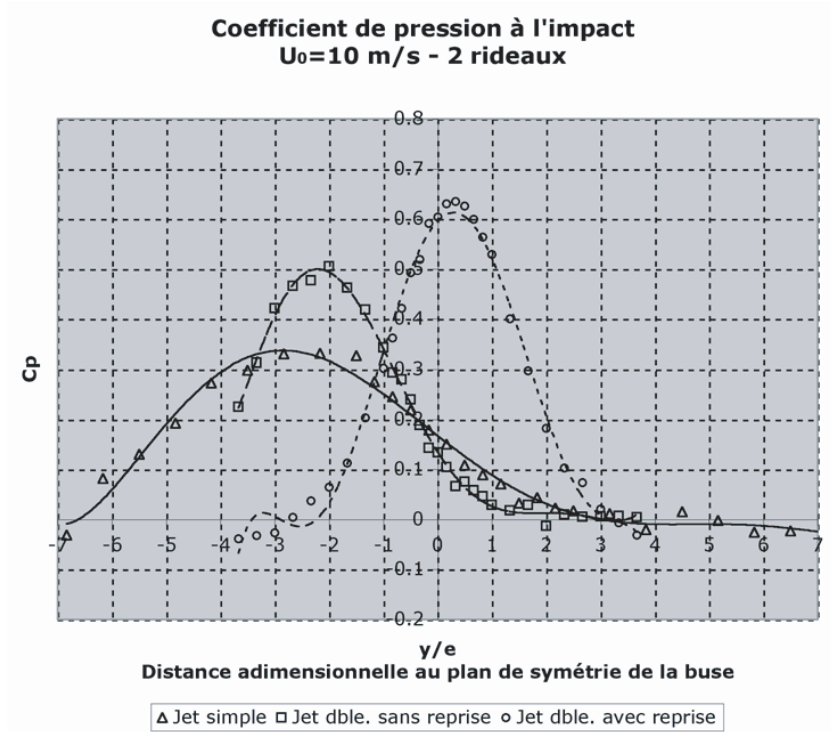

Fig. 12. Profils du coefficient de pression à l'impact de divers dispositifs à rideau d'air en configuration de confinement cellulaire. $C p=2\left(P-P_{\text {ext }}\right) / \rho U_{0}^{2}$.

\section{Conclusion}

Le contrôle des ambiances par rideaux d'air apporte des améliorations notables en économie d'énergie, maîtrise de la qualité des ambiances, sécurité du travail des manipulateurs, facilité d'intervention en cas de sinistre et isolement de zone polluée sans alimentation en air frais des foyers d'incendie...

Ce thème allie la technologie de mise en œuvre de flux d'air et la maîtrise de la structure dynamique des jets d'air impactant une paroi. L'application permet d'analyser le comportement du jet double flux avec reprise partielle dans la zone initiale d'établissement. 
Cette action a été un support pédagogique pour 3 groupes d'étudiants d'IUT sur deux années consécutives, la maquette « rideau d'air double flux »a été transportée à l'école des mines de Nantes, où des travaux proches étaient en cours. Les élèves ingénieurs ont pris le relais avec l'équipe d'enseignants chercheurs collaborant sur ce thème. Les travaux correspondants ont fait l'objet d'un travail pédagogique transdisciplinaire soutenu et primé par l'ANVAR dans le cadre des «Aides aux jeunes pour l'innovation ». Un stage de post-doctorant CNRS a permis de mettre en place les outils d'analyse nécessaires pour l'étude des structures cohérentes qui, à l'impact, contribuent aux transferts de masse. Une thèse sera prochainement soutenue sur le thème du confinement cellulaire; il sera ensuite envisagé d'intégrer la composante thermique en examinant le cas de situations anisothermes pour lesquelles un différentiel de température est imposé entre l'espace confiné et l'ambiance.

\section{Références}

[1] S. Gupta, Étude expérimentale du comportement dynamique et des performances de rideaux d'air en vue de la conception de systèmes de confinement cellulaire, Thèse de Doctorat, EMN-DSEE, Université de Nantes, 2005

[2] S. Gupta, M. Pavageau, C. Solliec, C. Rey, M. Dufresne de Virel, Particle Image Velocimetry (PIV) measurements in air-curtain systems designed for smoke confining in case of road tunnel fires, Int. Conf. Tunnel Safety and Ventilation., 2004, Graz

[3] L. Guyonnaud, Étude expérimentale de rideaux d'air Contribution à leur modélisation, Thèse de Doctorat, EMN-DSEE, Université de Nantes, 1998

[4] S. Maurel, C. Rey, C. Solliec, M. Pavageau, Caractéristiques cinématiques et structurelles d'un jet d'air plan turbulent frappant une plaque plane placée à distance variable, Mécanique et Industrie 5 (2004) 317-329

[5] M. Pavageau, E. Nieto, C. Rey, Odour and VOC confining in large enclosures using air curtains, Water Science and Technology 44(9) (2002)

[6] C. Rey, C. Cassin, Enveloppe Soleau n ${ }^{\circ}$ 40335, 29 mars 1999

[7] C. Rey, P. Maubert, Enveloppe Soleau nº 076083, 14 juin 2000

Retrouvez nos articles sur le site : www.edpsciences.org/meca 